\title{
The use of POD-DEIM model order reduction for the simulation of nonlinear hygrothermal problems
}

\author{
Tianfeng Hou ${ }^{1, *}$, Karl Meerbergen ${ }^{2}$, Staf Roels ${ }^{1}$ and Hans Janssen ${ }^{1}$ \\ ${ }^{1}$ KU Leuven, Department of Civil Engineering, Building Physics Section, Kasteelpark Arenberg 40, 3001 Heverlee, Belgium. \\ ${ }^{2}$ KU Leuven, Department of Computer Science, Numerical Analysis and Applied Mathematics section, Celestijnenlaan 200a, \\ 3001 Heverlee, Belgium.
}

\begin{abstract}
In this paper, the discrete empirical interpolation method (DEIM) and the proper orthogonal decomposition (POD) method are combined to construct a reduced order model to lessen the computational expense of hygrothermal simulation. To investigate the performance of the POD-DEIM model, HAMSTAD benchmark 2 is selected as the illustrative case study. To evaluate the accuracy of the POD-DEIM model as a function of the number of construction modes and interpolation points, the results of the POD-DEIM model are compared with a POD and a Finite Volume Method (FVM). Also, as the number of construction modes/interpolation points cannot entirely represent the computational cost of different models, the accuracies of the different models are compared as function of the calculation time, to provide a fair comparison of their computational performances. Further, the use of POD-DEIM to simulate a problem different from the training snapshot simulation is investigated. The outcomes show that with a sufficient number of construction modes and interpolation points the POD-DEIM model can provide an accurate result, and is capable of reducing the computational cost relative to the POD and FVM.
\end{abstract}

\section{Introduction}

The use of numerical simulations for building performance assessment is common, given that these allow a multitude of scenarios and factors to be evaluated smoothly. In building physics, hygrothermal models are often used to simulate the heat, air and moisture transfer in one- or multidimensional configurations: in literature we can find many computerbased tools for building performance prediction, usually based on standard methods for space and time discretization, such as Match, WUFI, Delphin or Hamfem [1-4]. These standard methods for space and time discretization can be very time consuming though, due to the strongly non-linear equations, the complex multi-dimensional spatial domains, and the long simulation intervals required. To lessen the computational expense, a faster surrogate model, which strongly reduces the calculation time of each deterministic simulation, is desirable.

Proper orthogonal decomposition (POD) has the potential to reduce the computational complexity and computational time for simulating a large complex system by approximating the high dimensional process by its "most relevant information", and it has been successfully applied in a variety of engineering fields, such as image processing, signal analysis, data compression, and recently equally in building physics [58]. Apart from these, Hou et al. [9] applied POD to reduce the calculation time for simulating building thermal performance. It is demonstrated that the POD model outperforms the conventional finite element model for simulating the wall thermal performance and that the use of POD to simulate a problem different from the training snapshot simulation can provide an accurate result. Hence the robustness of the POD method is confirmed. However, for nonlinear problems such as the highly nonlinear hygrothermal simulations, similar as the conventional simulation methods (for instance the finite element method and finite volume method), the inefficiency of POD arises from the high computational cost in repeatedly evaluating the nonlinear terms still in the scope of the dimension of the original problem [10]. To overcome this bottleneck, Chaturantabut et al. [10] proposed the discrete empirical interpolation method (DEIM). The main idea of DEIM method is to approximate the nonlinear term by combining projection with interpolation. DEIM projects the nonlinear terms onto a reduced basis and evaluates these terms at specific locations only, which are selected by a greedy algorithm. The component values at other locations can be interpolated through the reduced basis by an interpolation matrix that is pre-computed offline [11].

POD-DEIM has been successfully employed in many fields [12-15], and its potential to reduce the computational complexity of the nonlinear simulation has been addressed by Chaturantabut et al. [10]. However, to the best of the authors' knowledge, there is no prior work in the field of building physics giving a detailed investigation of the feasibility of POD-DEIM

\footnotetext{
* Corresponding author: tianfeng.hou $@$ kuleuven.be
} 
for building hygrothermal performance. Further, since the POD basis is constructed by the earlier results from a single simulation with the original model, a profound analysis of the robustness and flexibility of POD-DEIM for simulating problems different from the original configuration is crucial and should be illustrated extensively. In addition, as the computational complexity cannot entirely represent the computational cost, to provide a fair illustration of the superiority of PODDEIM over the conventional methods such as FVM, the accuracies of different approaches need to be compared, as a function of their calculation time. This paper targets these three research questions by applying POD-DEIM on a concrete building physical application, as well as analysing the robustness of POD-DEIM for simulating problems deviating from the original configuration. This paper is built up as follows. In section 2, a brief introduction of POD and POD-DEIM is given, focusing on their potential use for simulating hygrothermal performance. Further, in section 3, an introduction of the illustrative case study is provided and the performance of FVM, POD and POD-DEIM are compared based on their accuracy and computational time. The results are collected and discussed respectively. Finally, in section 4 our conclusion on the main findings on the potential of POD-DEIM is put forward.

\section{POD and DEIM for modelling building hygrothermal performance}

The hygrothermal performance of a building component can be assessed by analysing the transfer of heat and moisture through building materials, and it requires to get numerical simulation results for the coupled heat and moisture transport equation combined with information on the component geometry, its material properties and its boundary conditions. The conventional hygrothermal simulation models are typically based on numerical simulation methods applying a space and a time discretization, for instance, the FVM. The standard FVM for spatial discretization together with the common implicit Euler for temporal discretization to numerically solve a mass and energy conservative heat and moisture transfer equation yields a set of algebraic equations [4]:

$\left(C^{t+\Delta t, p}+\Delta t K^{t+\Delta t, p}\right) u^{t+\Delta t, p+1}=F^{t+\Delta t, p} \Delta t+C^{t+\Delta t, p} u^{t+\Delta t, p}$

$\Delta t K^{t+\Delta t, p} u^{t+\Delta t, p}-\left(S^{t+\Delta t, p}-S^{t}\right)$

where $\mathrm{p}$ indicates the iteration number, $u$ is the vector of unknown temperatures and capillary pressures at the spatial points, $\mathrm{K}$ denotes the stiffness matrix, $\mathrm{C}$ represents the mass matrix, $\mathrm{F}$ is the load vector and $\mathrm{S}$ are the conservation terms in relation to the mass and energy. Additionally, $\mathrm{t}$ and $\Delta \mathrm{t}$ respectively stand for the current time and the length of time step. The resulting system of algebraic equations is non-linear as the mass, stiffness and load matrices all depend on the unknown values of capillary pressure and temperature and hence a Newton-Raphson iterative scheme is implemented and the final resulting system of equations to be solved is: $\left(C^{t+\Delta t, p}+\Delta t K^{t+\Delta t, p}-\Delta t\left(d F^{t+\Delta t, p} / d u\right)\right) \Delta u^{t+\Delta t, p+1}=F^{t+\Delta t, p} \Delta t-$
$\Delta t K^{t+\Delta t, k} u^{t+\Delta t, p}-\left(S^{t+\Delta t, p}-S^{t}\right)$

\subsection{POD}

The introduction of proper orthogonal decomposition has been shortly presented by Hou et al. [9], with an application in the field of building thermal performance assessment. In this section we repeat the basic principles of POD and then extend its content and scope to building hygrothermal simulations. The proper orthogonal decomposition method was first proposed by Kosambi [16], and is also known as Karhunen - Loeve decomposition, principal component analysis, or singular value decomposition, and the connections of the methods are provided by Liang et al. [17]. The key idea of POD is condensing a high-dimensional process to its 'most relevant information' only. In this paper, that 'most relevant information' is extracted from earlier simulation results of the original numerical model through singular value decomposition (SVD). More specifically, the earlier simulation results are a set of data $u(x, t) \in R^{n \times m}$ containing all the results for all $n$ spatial points $x_{i}$ and all $m$ temporal points $t_{j}$, which is called the matrix of snapshots. Via SVD the snapshots matrix is decomposed into three distinct matrices, $u=\mathrm{B} \sum \mathrm{V}$, with $\mathrm{B} \in R^{n \times n}, \sum \in$ $R^{n \times m}$ and $\mathrm{V} \in R^{m \times m}$, as shown in Figure 1. Here, $B$ contains the set of orthonormal eigenvectors of $u u^{\mathrm{T}}$ and is hence called the matrix of construction modes, while $\sum$ is a rectangular diagonal matrix containing the singular values of $u$ in a reducing order on its main diagonal. Next, for the purpose of optimality only $k$ POD modes (here $k<n$ ) are selected and the snapshots matrix $u(x, t)$ is approximated with $\underline{\mathrm{B}}_{n \times k} \sum_{k \times k} \underline{\mathrm{V}}_{k \times m}^{\mathrm{T}}$ wherein $\underline{\mathrm{B}}_{n \times k}$, $\sum_{k \times k}$, and $\underline{\mathrm{V}}_{m \times k}$ are truncated versions of $\mathrm{B}, \sum$ and $\mathrm{V}$, as shown in Figure 2. Further, the truncated matrix of construction modes $\underline{\mathrm{B}}_{n \times k}$ is used to construct the reduced model for simulating new problems.

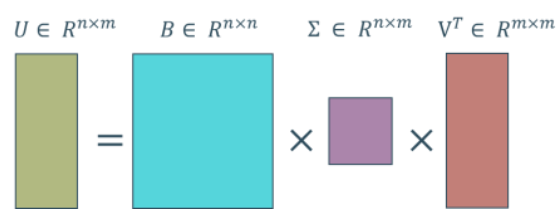

Fig. 1. Graphical concept of singular value decomposition.

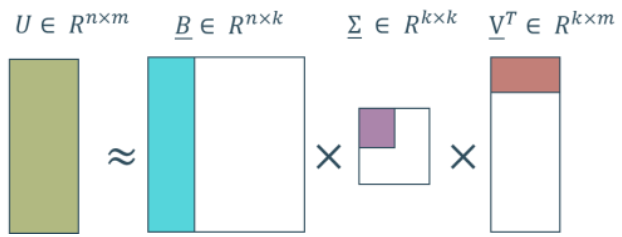

Fig. 2. Truncation matrices of $B, \sum$ and V.

Because $u$ is approximated by $\underline{\mathrm{B}}_{n \times k} \sum_{k \times k} \underline{\mathrm{V}^{\mathrm{T}}} k \times m$, we note $\underline{u}=\sum_{k \times k} \underline{\mathrm{V}}_{k \times m}^{\mathrm{T}}$, and thus $u \approx \underline{\mathrm{B}}_{n \times k} \underline{u}$. Then, by left multiplication of $\underline{\mathrm{B}}_{n \times k}^{\mathrm{T}}$ to (2), a reduced model is derived as follows: 
$\left(\underline{B}_{n \times k}^{T} C^{t+\Delta t, p} \underline{B}_{n \times k}+\Delta t \underline{B}^{T}{ }_{n \times k} K^{t+\Delta t, p} \underline{B}_{n \times k}-\Delta t \underline{B}_{n \times k}^{T}\right.$

$\left.\left({ }^{d} F^{t+\Delta t, p} / d u\right) \underline{B}_{n \times k}\right) \Delta \underline{u}^{t+\Delta t, p+1}=\underline{B}_{n \times k}^{T} F^{t+\Delta t, p} \Delta t-$

$\Delta t \underline{B}_{n \times k}^{T} K^{t+\Delta t, p} u^{t+\Delta t, p}-\underline{B}_{n \times k}^{T}\left(S^{t+\Delta t, p}-S^{t}\right)$

As a result, the new system (3) together with the relation $\mathrm{u} \approx \mathrm{Bn} \times \mathrm{ku}$ allows to approximate the solution at a reduced computational cost. Normally the lower the number of modes selected to build the reduced model, the lower the number of degrees of freedom contained in the reduced system, which hence leads to a lower computational expense. However, as mentioned in section 2.1, due to the nonlinearity of the mass, stiffness and load matrices, in each iteration step these nonlinear matrices need to be evaluated based on the updated values of the capillary pressure and temperature. To overcome this bottleneck and further reduce the computational expense, the DEIM technique is proposed.

\subsection{DEIM}

The main idea of DEIM is to approximate each nonlinear term by combining projection with interpolation. More specifically, DEIM projects the nonlinear terms onto a reduced basis and evaluates them at only several specific locations, which are selected by a greedy algorithm. The component values at other locations can be interpolated through the reduced basis by an interpolation matrix that can be pre-computed offline [11]. Wherein, the reduced basis related to the nonlinear terms are obtained by the POD method on their snapshots. A brief introduction of POD-DEIM is provided in the following. For the purpose of simplification, we denote the right hand side of equation (2): Ft $+\Delta t, p \Delta t-\Delta t K t+\Delta t$, put $+\Delta t, p-(\mathrm{St}+\Delta t, p$ - St) by $b$.

To reduce the computational cost of evaluating the nonlinear terms in each iteration step, we approximate these nonlinear components by projecting them onto a subspace. Suppose b can be projected on a subspace of dimension $\mathrm{m}$ and spanned by the basis $\mathrm{D}=\left[d_{1}, \ldots, d_{\mathrm{m}}\right] \in$ $R^{n \times m}$ as:

$$
b \approx D c
$$

where $\mathrm{c} \in R^{m \times 1}$ is the coefficient vector of the reduced basis. Since $n>m$ the system (4) is overdetermined. To determine $c$, we select $m$ distinct rows from (4) by multiplying a predetermined transpose of a boolean matrix $\mathrm{P} \in R^{n \times m}$ :

$$
P^{T} b=P^{T} D c
$$

By left multiplication of $\left(\mathrm{P}^{\mathrm{T}} \mathrm{D}\right)^{-1}$ to both sides of equation (5), the coefficient vector $\mathrm{c}$ can be derived:

$$
c=\left(P^{T} D\right)^{-1} P^{T} b
$$

and therefore (4), the approximation of $\mathrm{b}$ becomes:

$$
b \approx D\left(P^{T} D\right)^{-1} P^{T} b
$$

By left multiplication of $\mathrm{D}\left(\mathrm{P}^{\mathrm{T}} \mathrm{D}\right)^{-1} \mathrm{P}^{\mathrm{T}}$ to both sides of equation (2) we obtain:

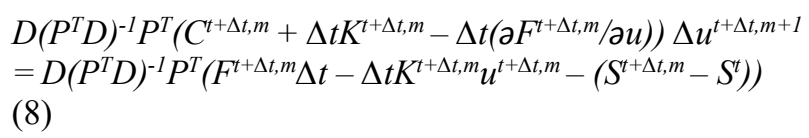

Combining the POD method, the POD-DEIM model reduction method transfers equation (3) to

$D\left(P^{T} D\right)^{-1} P^{T}\left(\underline{B}_{n \times k}^{T} C^{t+\Delta t, m} \underline{B}_{n \times k}+\Delta t \underline{B}_{n \times k}^{T} K^{t+\Delta t, m} \underline{B}_{n \times k}-\right.$
$\left.\Delta t \underline{B}_{n \times k}^{T}\left(d F^{t+\Delta t, m} / d u\right) \underline{B}_{n \times k}\right) \Delta u^{t+\Delta t, m+1}=D\left(P^{T} D\right)^{-1} P^{T}$
$\left(\underline{B}_{n \times k}^{T} F^{t+\Delta t, m} \Delta t-\Delta t \underline{B}_{n \times k}^{T} K^{t+\Delta t, m} u^{t+\Delta t, m}-\right.$
$\left.\underline{B}_{n \times k}^{T}\left(S^{t+\Delta t, m}-S^{t}\right)\right)$

The transpose of boolean matrix PT guarantees that each nonlinear term of system (3) only needs to be evaluated at specific locations, while other component values are interpolated by $\mathrm{D}\left(\mathrm{P}^{\mathrm{T}} \mathrm{D}\right)^{-1}$ that can be precomputed offline. In addition, the projection basis $\mathrm{D}$ is calculated by applying POD on the nonlinear snapshots of $b$, and the interpolation indices which are the entries of the boolean matrix $\mathrm{P}$ are derived from the DEIM algorithm proposed by Chaturantabut et al. [10].

\section{Application case study}

In this section, a set of analyses are performed to provide an exhaustive evaluation of the performances of the POD-DEIM model order reduction method, more specifically the accuracy and computational cost of POD-DEIM are respectively compared with FVM and POD. To do so, a reference benchmark which can assess the performance of different simulation models is required. In this paper the HAMSTAD benchmark 2 [18] is selected. This case study analyses the isothermal moisture redistribution in one material layer caused by sudden drops of the relative humidity at both surfaces. The thickness of the layer is $200 \mathrm{~mm}$ and the simulation period is 1000 hour. Initial relative humidity is constant and the layer is in moisture equilibrium with ambient air. Detailed information about HAMSTAD benchmark 2 its construction geometries, material properties, boundary conditions - can be found in [18]. The case study is simulated respectively by FVM, POD and PODDEIM, and all solvers necessary for the purpose have been developed by the first author. The accuracy of the obtained solutions is then evaluated by comparison with a reference solution from a FVM calculation with 200 spatial mesh elements and a fixed time step of one hour.

As stated in section 1, the reduced model obtained by POD and POD-DEIM is usually used for simulating problems with different configurations/or for longer simulation periods. Therefore, in this section, two research issues are studied: 1. the accuracy and computational cost of FVM and POD and POD-DEIM constructed with the full set of snapshots are compared. 2. the performance of POD and POD-DEIM for simulating problems with longer simulation intervals is assessed. The results are respectively put forward in sections 3.1 and 3.2. In order to compare the overall behavior of different models, the deviation of solutions of different models relative to the reference solution are quantified with the average absolute moisture content error (equation (10)): 
$\sum_{I \leq i \leq N x, I<j<N t} \mid u_{\text {reference }}(i, j)$ - $u_{\text {reduced }}(i, j) \mid /\left(N_{x} \times N_{t}\right)$

Here, $u_{\text {reference }}$ and $u_{\text {reduced }}$ are respectively the moisture content results of the reference solution and the reduced model with $N_{x}$ and $N_{t}$ the number of spatial and temporal discretization points of different methods.

\subsection{FVM and POD and POD-DEIM constructed by the full set of snapshots}

In this section, the calculation accuracy and computational cost of FVM and POD and POD-DEIM constructed by the full set of snapshots are judged. For getting a direct view of the performance of different models, we select the moisture content profiles at 100 hours as target output. The profile is simulated by the three models with different number of modes/nodes and interpolation points. More specifically, the FVM models are constructed by 5 and 50 nodes. The POD models are constructed by 5 and 10 modes, and for each POD model 50 interpolation points are used to build the POD-DEIM models. For the FVM, in order to obtain moisture content values at the spatial points of the reference solution, the intermediate values are calculated by interpolation. All profiles together with the result of the reference solution are shown in Figure 3. Further, to obtain a comprehensive view of the performance of different models, the average absolute moisture content deviations, see equation (10), of different models are compared and the results are shown in Figure 4. More specifically, the FVM and POD models are constructed by $5,10,15,20,25$ nodes/modes. For each POD models, different number of interpolation points (65 points, 55 points, 45 points, 35 points, 25 points) are used to build the POD-DEIM models. Finally, as the number of modes/nodes and interpolation points cannot entirely represent the computational cost of different models, to provide a fair comparison of the computational performance of different approaches, the average moisture content differences are again presented in Figure 5, but now as a function of the calculation time. More specifically, the FVM models are constructed with 80 to 150 nodes, with an interval of 5 nodes. The POD models are constructed with 4 to 18 modes. In relation to the POD-DEIM models, 7, 10 and 25 POD modes are used together with 40 to 44 interpolation points.

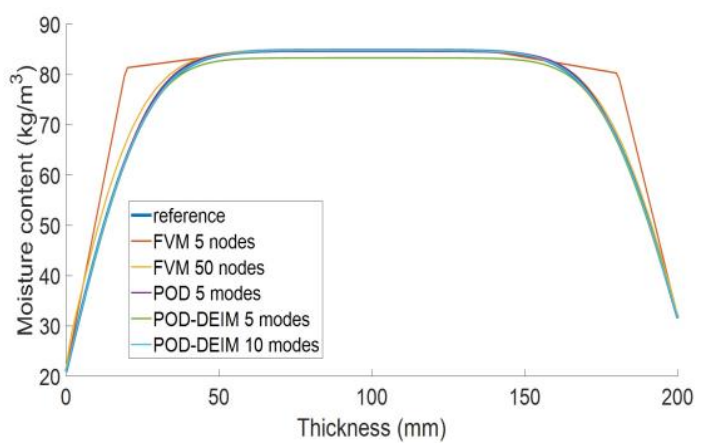

Fig. 3. Moisture content profiles at 100 hours of the reference solution and solutions of FVM models and reduced models constructed by different number of nodes/modes.

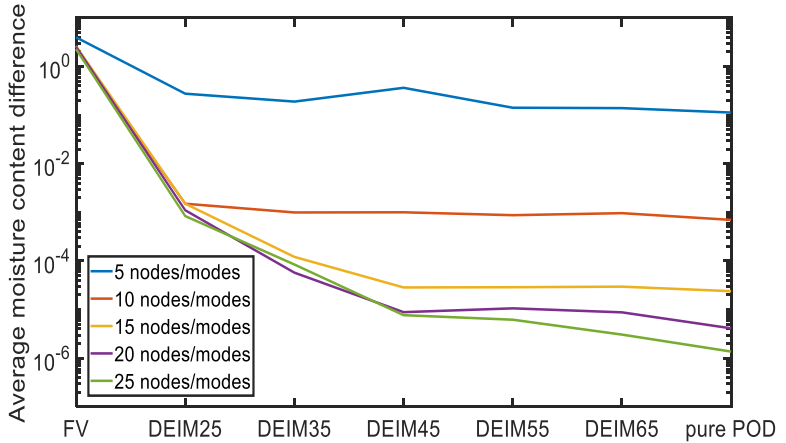

Fig. 4. Average moisture content difference between the reference solution and solutions of FVM models and reduced models constructed by different number of nodes/modes and interpolation points.

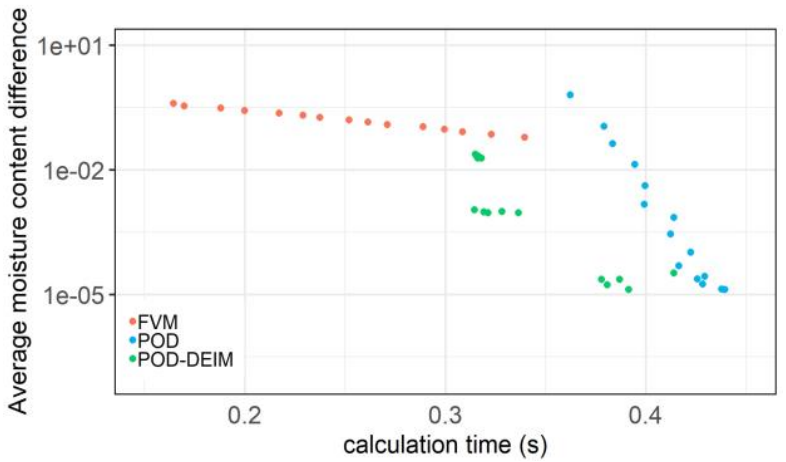

Fig. 5. Average moisture content differences between the reference solution and solutions of the FVM, POD and PODDEIM models, as a function of the calculation time.

Figure 3 shows that for the POD model with 5 modes and POD-DEIM model with 10 modes there is no observable differences between the reference solution and the reduced models' approximations. Hence, for HAMSTAD benchmark 2, with a relatively small number of construction modes and interpolation points, both POD and POD-DEIM can provide an accurate result of the moisture distribution in the material layer. On the other hand, a rather large differences can be found between the FVM model with only 5 nodes and the reference solution.

Figure 4 presents an overview of the accuracies of different models constructed by different number of POD modes and interpolation points. It confirms the results of Figure 3, with a sufficient number of construction modes and interpolation points, POD-DEIM can provide an accurate result and the accuracy of the POD-DEIM method increases as the amount of their construction modes and interpolation points raises. On the other hand, Figure 4 also shows that with more than 45 DEIM points the accuracies of the reduced models do not increase significantly.

Figure 5 finally confronts the obtained calculation accuracies with the required computation times. This demonstrates that at large inaccuracies (moisture content errors around $0.1 \mathrm{~kg} / \mathrm{m}^{3}$ ) the FVM is the most efficient. At better accuracies (moisture content errors below 0.01 $\mathrm{kg} / \mathrm{m}^{3}$ ) on the other hand, POD-DEIM is obviously the 
most efficient method, since it allows to obtain the best accuracy at a given computation time, strongly outperforming FVM and POD. It should be mentioned that, in this paper all the POD and POD-DEIM models are based on the snapshots of the reference solution, and hence the deviation of solutions of POD and POD-DEIM relative to the reference solution tends to get very small. We also investigated the performance of POD and PODDEIM models that are derived from simulation deviating from the reference solution. As the results hereof are similar to Figure 5, they are not explicitly presented in the paper.

\subsection{POD-DEIM for longer simulation intervals}

In section 3.1, it was shown that the POD-DEIM model based on the full set of snapshots can provide an accurate result when simulating the same system. However, in practice this recalculation of the same problem does not add any value, as all the information is already included in the matrix of snapshots. Instead, in this section we are investigating the use of POD-DEIM to construct a reduced model for simulating different problems in relation to longer simulation period. In other words, the accuracies of the POD-DEIM models constructed by different size of snapshots are evaluated. More specifically, for getting a more direct view of the performance of POD-DEIM as a function of the size of its snapshot, similar as before the moisture content profiles of the building component simulated by POD and POD-DEIM constructed by 18 modes and different size of snapshots (10 hours and 30 hours) are shown in Figure 6. Here, all the POD-DEIM models are built with 50 interpolation points. In addition, to further evaluate the accuracy of POD and POD-DEIM methods as a function of the amount of the construction modes, the average moisture content differences between the reference solution, POD and POD-DEIM models built by different size of snapshots, as a function of the amount of their construction modes are shown in Figure 7. Here, all the POD-DEIM models are built with 50 interpolation points.

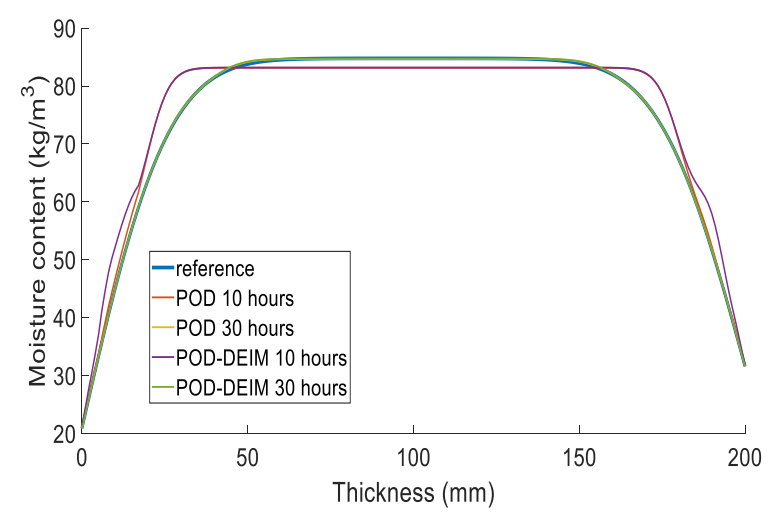

Fig. 6. Moisture content profiles at 100 hours of the reference solution and solutions of the reduced models constructed by different size of snapshot.

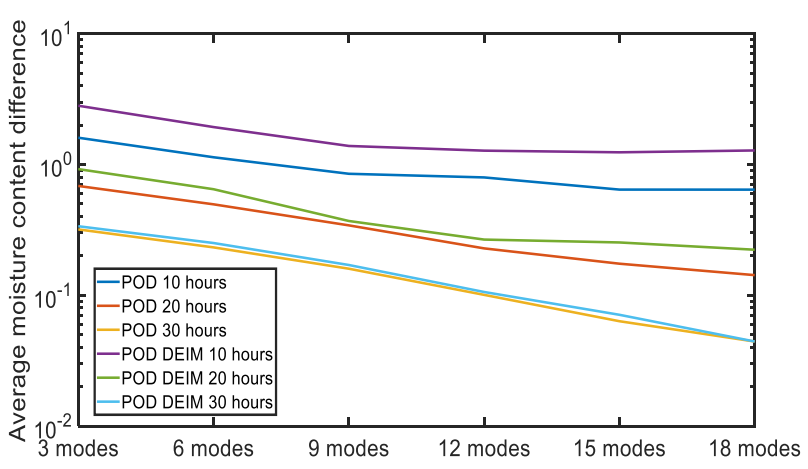

Fig. 7. Average moisture content difference between the reference solution and solutions of the reduced models constructed by different size of snapshots and different number of construction modes.

Figure 6 is similar to Figure 1, but now for different size of snapshots. It is demonstrated that, with 30 hours snapshot and 18 modes, the moisture distribution in the material layer can be obtained rather accurately by both POD and POD-DEIM models. On the other hand, larger differences can be found between the reduced models with only 10 hours snapshot and the reference solution.

Figure 7 again illustrates that the accuracies of POD and POD-DEIM increase as the size of their snapshots raises. It is also shown that, with a sufficient size of snapshots (from simulation intervals of 30 hours), the accuracies of the POD and POD-DEIM models increase as the number of their modes raises. On the other hand, when the size of snapshots is insufficient (from simulation of 10 hours), an inaccurate result (moisture content errors larger than $1 \mathrm{~kg} / \mathrm{m}^{3}$ ) may be obtained. As result, this confirms the final observation of the section above, indicating that with only a 30 hours of actual standard calculation, suffice to reproduce the long-term moisture content behaviour of the building component.

\section{Conclusion}

In this paper, we investigated the potential of PODDEIM for the simulation of building hygrothermal performance based on a case study of HAMSTAD benchmark 2. It is shown that (see Figures 3 to 5) the accuracy of POD-DEIM increases as the number of the construction modes and interpolation points raises. With a rather small number of construction modes and interpolation points, POD-DEIM is capable to provide an accurate result. In combination with computation time though, the POD-DEIM method is more efficient than FVM and POD. Further, the use of POD-DEIM to simulate a problem different from the training snapshot simulation is investigated. It is shown that (see Figures 6 and 7), the accuracy of POD-DEIM increases as the size their snapshots raises, and with a sufficient size of snapshot POD-DEIM is able to reproduce the long-term moisture content behaviour of the building component.

This work was supported by the European Union's Horizon 2020 research and innovation program under grant agreement No 637268 (RIBuild). 


\section{Reference}

1. Grunewald J, Doctoral dissertation, TU Dresden, (1997).

2. Künzel, H.M, Doctoral dissertation, Universität Stuttgart, (1994).

3. Pettersen, T.D, ENERG BUILDINGS, 21, (1994).

4. Janssen, H., Blocken, B. and Carmeliet, J, Int. J. Heat Mass Transf, 50, (2007).

5. Li, J., Liu, J., Cao, X. and Jiang, N, ENERG BUILDINGS, 127 (2016).

6. Meyer, R.D. and Tan, G, ENERG BUILDINGS, 73, (2014).

7. Wei, Y, Zhang, T.T. and Wang, S, BUILD ENVIRON, 96, (2016).

8. Tallet, A., Liberge, E. and Inard, C, BUILD SIMUL-CHINA, 10, (2017).

9. Hou,T., Janssen, H. and Roels, S, ENERG BUILDINGS, (to be published).

10. Chaturantabut S, Sorensen D.C, SIAM J SCI COMPUT 32 (2010).

11. Tiso P., Rixen D.J. 2013, Topics in Nonlinear Dynamics, 1 (2013).

12. Ştefănescu, R. and Navon, I.M, J. Comput. Phys, 237 (2013).

13. Chaturantabut, S. and Sorensen, D.C, MATH COMP MODEL DYN, 17 (2011).

14. Ştefănescu, R., Sandu, A. and Navon, I.M, J. Comput. Phys, 295 (2015).

15. Yang, $H$. and Veneziani, A, APPL NUMER MATH, 115, (2017).

16. Kosambi, D.D., 2016. Statistics in function space. In DD Kosambi (pp. 115-123). Springer, New Delhi.

17. Liang Y.C., Lee H.P., Lim S.P., Lin W.Z., Lee K.H., Wu C.G, J SOUND VIB 252, (2002).

18. Hagentoft C.E. 2002. Hamstad wp2-benchmark package. Technical report, Dept. of Building Physics-Chalmers University of Technology. 